\title{
In situ observation of syntactic foams under hydrostatic pressure using X-ray tomography
}

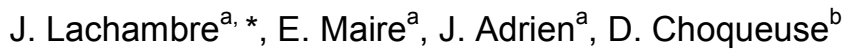

\author{
a Matériaux: Ingénierie et Science, UMR CNRS no. 5510, Batiment St Exupery, INSA Lyon, 69621 Villeurbanne \\ Cedex, France \\ ${ }^{\mathrm{b}}$ Materials \& Structures Group (RDT/MS), IFREMER Centre de Brest, BP70, 29280 Plouzané, France \\ *: Corresponding author: Joël Lachambre, tel.: +33 472437176 ; fax: +33 472438539 ; \\ email address : joel.lachambre@insa-lyon.fr
}

\begin{abstract}
:
Syntactic foams (hollow glass microspheres embedded in a polymeric matrix) are being used increasingly for the purpose of thermal insulation in ultradeep water. A better understanding of the damage mechanisms of these materials at the microsphere scale under such a hydrostatic loading condition is of prior importance in determining actual material limits, improving phenomenological modelling and developing novel formulations in the future. To achieve this goal, a study based on X-ray microtomography was performed on two syntactic foam materials (polypropylene and polyurethane matrix) and a standard foamed PP. A special set up has been designed in order to allow the X-ray microtomographic observation of the material during hydrostatic pressure loading using ethanol as the pressure fluid. Spatial resolution of $(3.5 \mu \mathrm{m})^{3}$ and in situ non-destructive scanning allowed a unique qualitative and quantitative analysis of the composite microstructure during stepwise isotropic compression by hydrostatic pressure up to $50 \mathrm{MPa}$. The collapse of weaker microspheres were observed during pressure increase and the damage parameters could be estimated. It is shown that the microspheres which are broken or the porosities which are close to the surface in the foamed PP are filled by a fluid (either the ethanol or the polymeric matrix itself). The hydrostatic pressure decreases the volume of the foam only slightly. In the PU matrix, ethanol diffusion is seen to induce swelling of the matrix, which is an unexpected phenomenon but reveals the high potential of X-ray microtomographic observation to improve diffusion analysis in complex media.
\end{abstract}

Keywords: Syntactic foams ; X-ray tomography ; Hydrostatic compression test ; Non-destructive testing ; Synchrotron radiation

\section{Introduction}

Syntactic foams, which are made of hollow glass microspheres embedded in a polymer matrix, are becoming more widely used for thermal insulation of offshore pipelines and bundles which convey oil and gas resources from extraction sites located in deep water [1], [2], [3], [4], [5], [6], [7], [8], [9], [10], [11] and [12]. Insulation material durability contributes directly to the maintenance of reliable offshore production (flow assurance) over 20 years of service. The combined effects of increasing hydrostatic pressure (30 MPa at a depth of $3000 \mathrm{~m}$ ) induced by the increasing depth of water, temperature gradient and degradation due to water contact lead to more stringent requirements for the properties of these syntactic composite materials. Therefore, a better understanding of syntactic foam degradation mechanisms in extreme conditions is necessary to determine the actual limits of materials and assess their lifespan, to improve phenomenological modelling of long-term behaviour and to develop novel formulations in the future.

Recently, investigations by means of high-resolution synchrotron X-ray tomography have been performed in order to characterise the microstructure of such materials in the dry state and after immersion in water [13] 
and [14]. This allowed very fine details such as the thickness of the hollow spheres, their size distribution and the volume fraction of the three components (gas, glass and polymer) to be quantified. Tests after hydrostatic pressure loading were also performed on polymeric foams [15] and on syntactic foams [13], but in all these studies the foams were always studied ex situ.

The hydrostatic compression of syntactic foams in situ in tomography has never been carried as far as the author is aware. Confined uniaxial dry compression in situ tests have already been reported in [16]. The results of these tests show that the compression axis is a preferred direction regarding the occurrence of damage. Considering the edge effects and the damageable behaviour of the material, the confined uniaxial compression leads to a non homogeneous loading state of the material. In order to be close to the "in service" loading conditions for syntactic foams, the present paper focuses on isostatically constrained compression in a pressurized liquid. This loading mode is applied in situ during tomographic observation to analyse the collapse mechanisms occurring in two different syntactic foams with polymer matrices, namely polypropylene $(\mathrm{PP})$ and polyurethane (PU) and a foamed polypropylene. This non-destructive technique is shown to be a very powerful tool to characterize the microstructure of cellular materials during in situ mechanical tests. The compression behaviour of the three kinds of foams is compared in terms of local damage events, swelling of the matrix and the fraction and the morphology of filled-in spheres.

\section{Materials and experimental techniques}

\subsection{Materials}

Three types of foams were used in the present study. Two syntactic foams which were made of glass microspheres (type S38 from 3M) with two different types of matrix material: Glass Syntactic Polypropylene GSPP and Glass Syntactic Polyurethane GSPU; and a "classic" foam, which did not contain any hollow microspheres, just porosities. The solid part of this last foam, MPP, was made of polypropylene. Syntactic foams are made by mixing liquid ingredients (PU or PP) with the glass microspheres under a vacuum. The resulting slurry is then injected into a mold. Table 1 shows the formulation and reference of these three materials and their physical and mechanical properties measured by the hydrostatic compression test developed by Choqueuse et al. in [11]. This method is based on the loss of buoyancy under hydrostatic compression which is directly linked with the filling of microspheres and gives access to an estimation of the maximum service pressure.

\begin{tabular}{ccccccc}
\hline ref & type & $\begin{array}{c}\text { Matrix } \\
\text { material }\end{array}$ & $\begin{array}{c}\text { Density } \\
\left(\mathrm{kg} \cdot \mathrm{dm}^{-3}\right)\end{array}$ & $\begin{array}{c}\text { M atrix } \\
(\% \text { vol })\end{array}$ & $\begin{array}{c}\text { Gas } \\
(\% \text { vol })\end{array}$ & $\begin{array}{c}\text { M ax service } \\
\text { pressure (M Pa) }\end{array}$ \\
\hline GSPP & syntactic foam & polypropylene & 0.64 & 50 & 43 (2) & 30 \\
GSPU & syntactic foam & polyurethane & 0.85 & 75 & 22 & 20 \\
MPP & foam & polypropylene & 0.79 & 87 & 13 & 12 \\
\hline
\end{tabular}

(1) extra volume corresponds to glass volume

(2) $2 \%$ volumetric of gas not included in the glass bubble

Table 1: materials and their properties.

\subsection{X-ray tomography}

The internal observation of damage during the compression tests was carried out by means of a threedimensional (3D) non-destructive technique, high-resolution X-ray tomography. Using this technique, disturbance-free information about the microstructure throughout the volume could be obtained. Very detailed descriptions of this technique can be found in Refs. [17, 18]. The important features of this characterization method are briefly summarized here. Tomography combines information from a large number of X-ray radiographs taken with different viewing angles of the sample. The technique contains a computed 
step, i.e., it includes a recalculation step during which a 3D map of the local absorption coefficient in each elementary volume of the sample is retrieved from the set of absorption radiographs. This map gives an indirect image of the microstructure. In the present study, the samples were scanned using a high-resolution X-ray tomograph located at the ESRF (beam line ID 19) in Grenoble (France). X-ray tomography was performed at a voxel size of $(3.5 \mu \mathrm{m})^{3}$. The energy was set to $17.5 \mathrm{keV}$. The distance between the sample and the detector governed by the size of the compression rig (see figure 1) was $\sim 50 \mathrm{~mm}$. A set of 700 projections was taken within $180^{\circ}$. The acquisition time was about 5 minutes. The detector was a CCD camera with $1024 \times 512$ (binning mode) sensitive elements coupled with a scintillator.

\subsection{Hydrostatic compression}

Hydrostatic compression tests were carried out at room temperature by means of a special isostatic pressure rig schematized in fig. 1 and placed in a compression device described in [17]. The sample (size about $2 \times 2 \times 2 \mathrm{~mm}^{3}$ ) was glued onto a little support in a chamber filled with ethanol. A piston compressed the fluid which applied a hydrostatic compression on the sample. The piston was driven with a small compression rig [17] set on the rotation stage of the tomograph. The chamber was made of an aluminium tube of $6 \mathrm{~mm}$ in diameter with a thickness of $0.9 \mathrm{~mm}$. Aluminium was chosen for its good ratio between $\mathrm{X}$-ray transparency and pressure resistance capability.

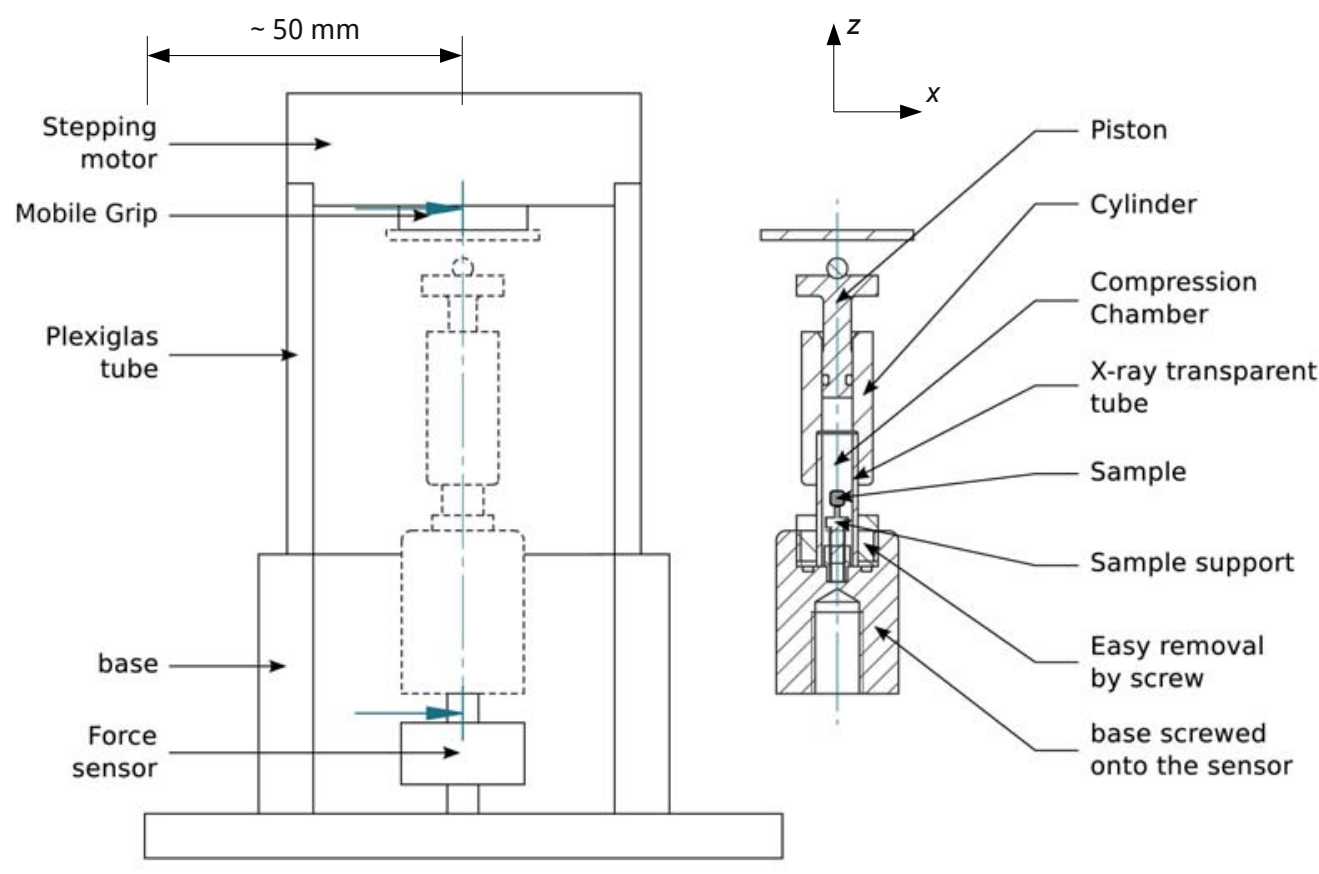

Figure 1: diagram of the special isostatic pressure rig on the right and the compression device where it is placed on the left.

\section{Experimental protocol}

Ethanol was chosen here as the isostatic fluid for safety reasons because it is likely to be more stable than water under X-Ray irradiation. Indeed, water can boil under high intensity X-rays so this could disrupt the acquisition and the reconstruction could fail. Three kinds of scans were made for each foam sample: a first scan without the pressure envelope to observe the material in its initial state, a second scan in ethanol and finally a set of several scans at increasing pressures. The pressure was applied step by step. For each 


\begin{tabular}{cccc}
\hline Scan number & M PP & GSPP & GSP U \\
\hline 1 & 0 & 0 & 0 \\
2 & 55 & 83 & 115 \\
3 & 104 & 355 & 255 \\
4 & 133 & 500 & 343 \\
5 & - & - & 535 \\
\hline
\end{tabular}

Table 2: pressure (in bars) applied during the different steps to the three samples.

step, we waited about 5 minutes until the pressure stabilized before acquiring each scan. Table 2 shows the pressure levels for which X-ray tomoghraphy scans were performed for the 3 samples.

For $50 \mathrm{MPa}$, the displacement of the piston was about $2 \mathrm{~mm}$. The corresponding compressed volume is $56.5 \mathrm{~mm}^{3}$, as the volume chamber approximates $1000 \mathrm{~mm}^{3}$, this corresponds to a $5.65 \%$ of volumetric compression. Takiguchi and Uematsu [19] show that for a temperature of $40{ }^{\circ} \mathrm{C}$ (one can assume that the temperature of the fluid during compression and scanning was close to this temperature) the volume variation of ethanol between $0.1 \mathrm{MPa}$ and $50 \mathrm{MPa}$ is $4.62 \%$. This means that the displacement observed is mostly the result of the compressibility of the ethanol and only slightly due to the compression of the sample.

\section{Qualitative observation}

\subsection{Initial state}

The reconstruction of a slice in the initial state of each material shows the general aspect of the foams (figures 2a, 4a and 5a respectively for GSPP GSPU and MPP foam). The two syntactic foams are more or less similar but the GSPU appears to have smaller spheres, the foam was provided as a plate that was cut in $2 \mathrm{~mm}$ edge cubes with a cutter. The cut is not clean and pre-damaged regions can be observed close to the sample's surfaces in the form of microsphere pull out and micro-cracks creation. For the GSPU sample, one side is very straight and has no traces of pulling out. This is because it is a side initially in contact with the mould. The third foam is very different, porosity is large comparatively to the size of the sample so distribution is not very homogeneous.

\begin{tabular}{lllr}
\hline Foam & Type of inclusion & M atrix & \% of porosity \\
\hline GSPP & Microsphere & Polypropylene & $21.21 \%$ \\
GSPU & Microsphere & Polyurethane & $18.54 \%$ \\
MPP & Porosities & Polypropylene & $5.92 \%$ \\
\hline
\end{tabular}

Table 3: porositiy measured using tomographic images.

Table 3 shows the amount of air present in each sample measured using these initial images. This amount seems to be slightly smaller than what could be expected from the composition of the foam (see table 1). In the case of MPP foam, the cavities are big $(400 \mu \mathrm{m})$ compared to the resolution of the tomograph so the measurement of their amount can not reasonably be questioned. The discrepancy is probably partly due to the small size of the samples $\left(2^{3} \mathrm{~mm}^{3}\right)$ investigated in X-Ray tomography so the amount of porosity can be locally smaller than the average value in a bigger sample (the original as-processed sample from where is cut the smaller one is about $100^{3} \mathrm{~mm}^{3}$ ). In the cases of GSPP and GSPU, the dimension of the porosity is rather small compared to the resolution of the tomograph used. Consequently, an error of one pixel in 
the measurement of the radius of each cavity can typically lead to a $10 \%$ change (increase or decrease) in the measured volume fraction. Note also that because of the presence of a complex set of phase contrast fringes at the periphery of each sphere, the exact radius is very difficult to threshold with precision. This could explain the observed discrepancy.

\subsection{Deformed state}

\subsubsection{Foam GSPP}

The principal mechanism of the disappearance of porosity is shown in figure 2. A particular microsphere is highlighted with a arrow in the initial state (fig. 2a), and it is always present after immersing in ethanol (fig. 2b). At 83 bars, the microsphere is filled with a fluid (fig. 2c). From the present observation, it is not possible to determine if this fluid is the polymeric matrix or if it is ethanol because these two constituents absorb X-rays in the same manner. This observation is discussed later on in the paper. The glass shell of the microsphere is then probably cracked, but because loaded isotropically, it remains in place, and a fluid is observed to fill the sphere.

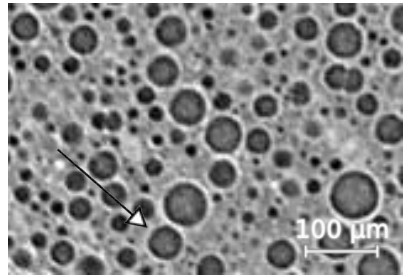

(a) in air

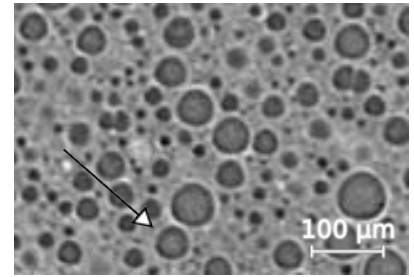

(b) in ethanol

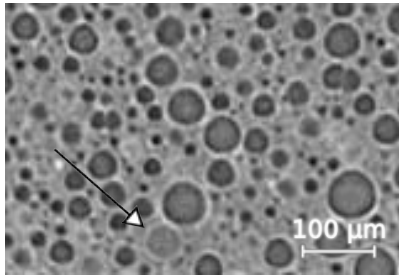

(c) under 83 bars

Figure 2: disappearance of a microsphere in GSPP.

In some cases, the microsphere doesn't fill in one single step (figure 3). In this second region of the same sample, the filling starts between 83 bars and 355 bars but it is not completed at 355 bars. At 500 bars the sphere is finally observed to be completely filled. The few compression steps don't allow us to witness the details of the filling in, but we can assume from this observation that it is not perfectly instantaneous.

\subsubsection{Foam GSPU}

The same filling processes are observed in the GSPU foam (figure 4). Some microspheres, probably in contact with the surface of the sample, disappear immediately after immersion in ethanol (fig. 4b), others after 115 bars (fig. 4c) and after 343 bars (fig. 4d).

A swelling of the matrix is also clearly observed as illustrated in figure 4 . The centre of some easily recognizable microspheres is highlighted for the purpose of visualization by a little circle in the figures so these allow us to follow the evolution of the distance between spheres in the sample. The evolution of the spacial position of these circles is shown in figure 4e; black circles represent the initial states, the dark gray ones represent the immersed state, the light grey and the white ones respectively represent 115 bars and 343 bars. The four reconstructed slices (not only the region of interest) used for this graph are adjusted to coincide with the centre of the foam specimen (see figure $2 \mathrm{f}$ ).

As the volume of microspheres doesn't increase, the swelling is due to the ethanol uptake of the PU matrix in the sample. Swelling is already important just after ethanol immersion. It then increases with the applied pressure either because pressure forces more ethanol into the matrix or because time allows for more ethanol to swell the matrix. It should also be noticed that while some spheres are filled with ethanol, some others remain empty during the entire test. It would seem that the filling spheres are rather large. This supports the results found in [16] showing that the larger spheres collapse at lower stress during constrained compression. 


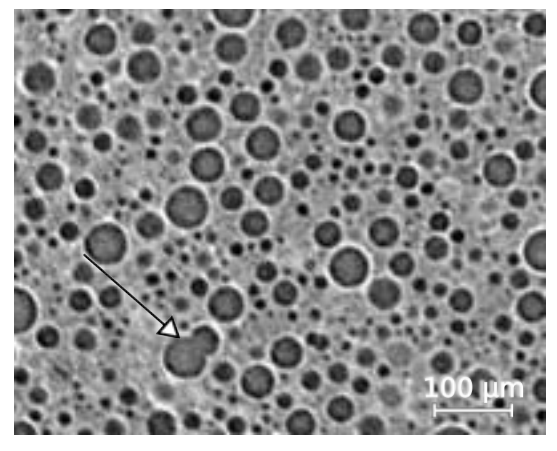

(a) in air

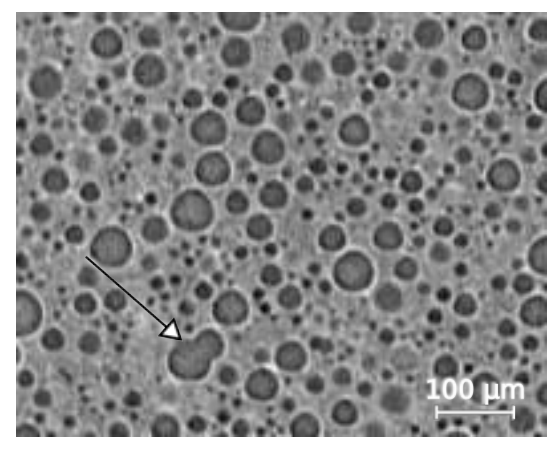

(b) in ethanol

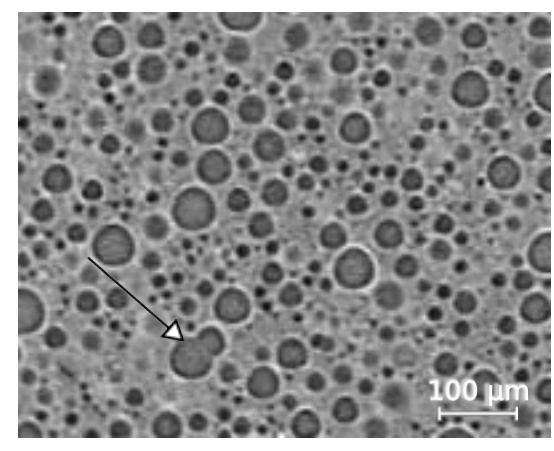

(c) under 83 bars

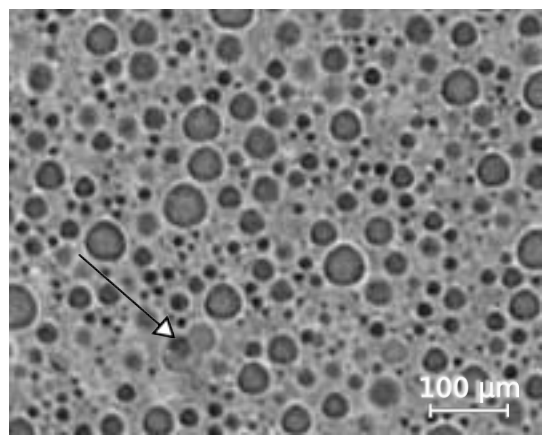

(d) under 355 bars

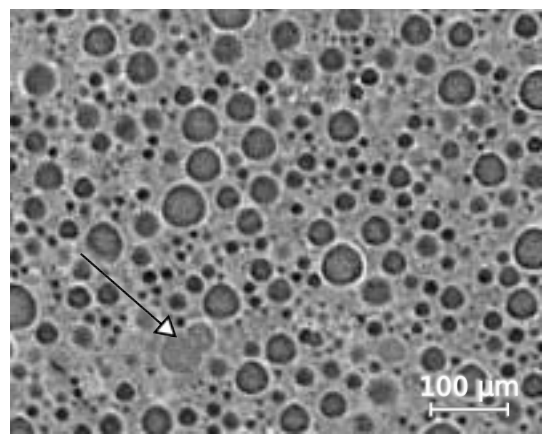

(e) under 500 bars

Figure 3: collapse of some microspheres in GSPP foam. 


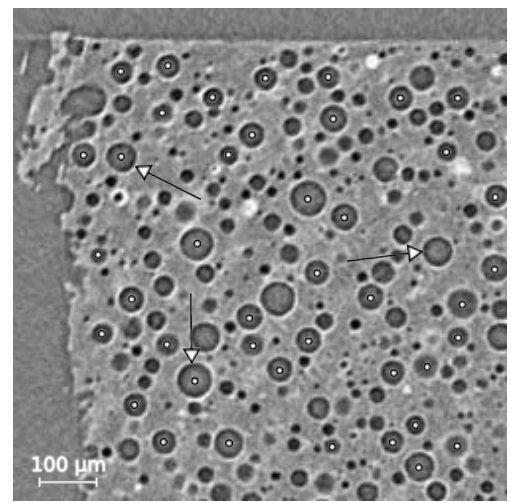

(a) in air

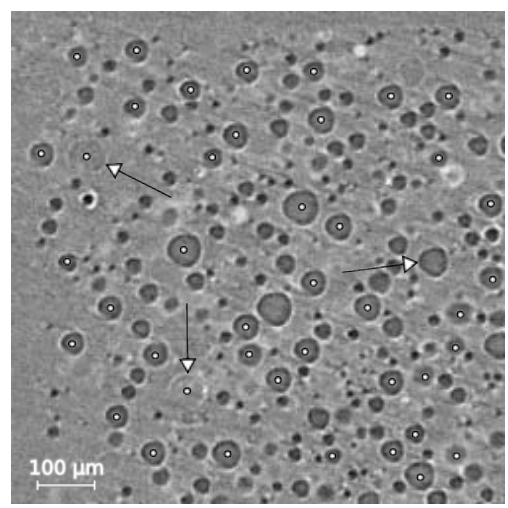

(c) under 115 bars

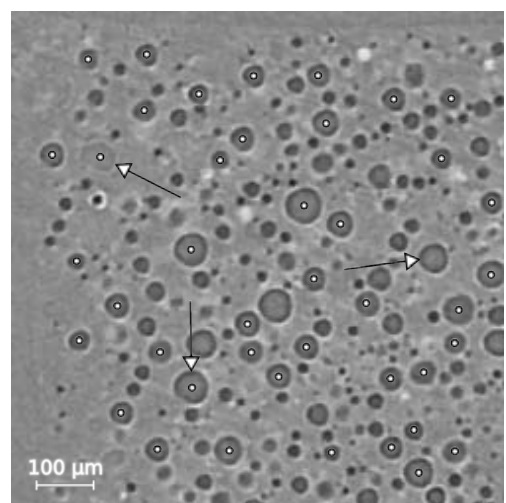

(b) in ethanol

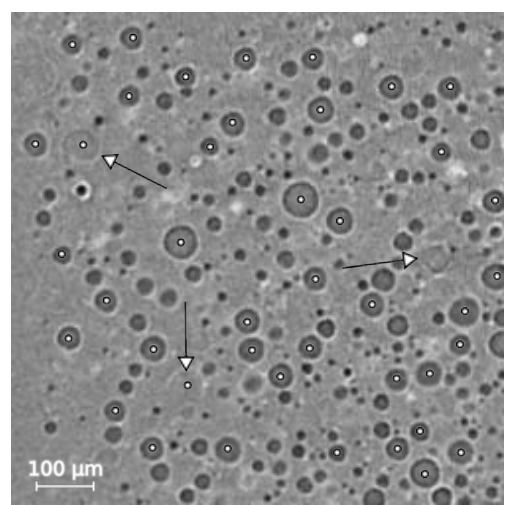

(d) under 343 bars

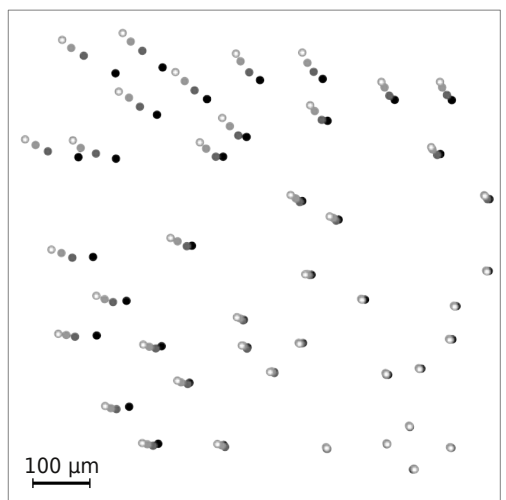

(e) evolution of position for some microspheres

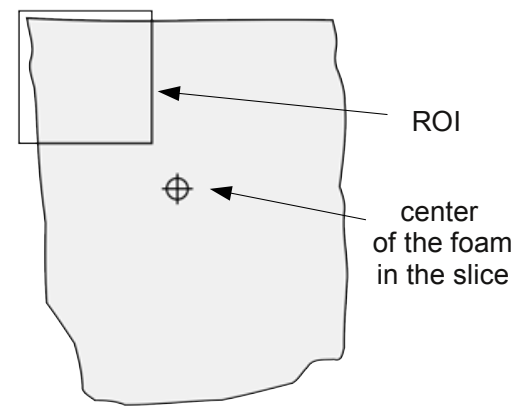

(f) region of interest (ROI)

Figure 4: disappearance of microspheres in GSPU foam and swelling due to the ethanol uptake of the matrix. 


\subsubsection{Foam $M P P$}

The third foam is not syntactic like the two others. As porosity is great compared to the sample size. Figure 5 shows the evolution of a typical slice before immersion and under hydrostatic pressure at 104 bars. In this foam, there is no glass, so the gas is not confined. If one examines carefully figure $5 \mathrm{~b}$, a slight outline which looks like the disappeared porosity can be distinguished by the human eye on the left of the figure. A third figure (5c) was produced by stacking the 104 bars state figure with the outline created with the initial state figure. Although not easy to show using a numeric image, we certify that under pressure, the disappeared porosities are still here, filled with ethanol. Some of these porosities are close to the surface but some are not. It can also be observed from the comparison of the outline in figure $5 \mathrm{c}$ that the size of the large remaining porosities is significantly reduced due to the applied pressure.

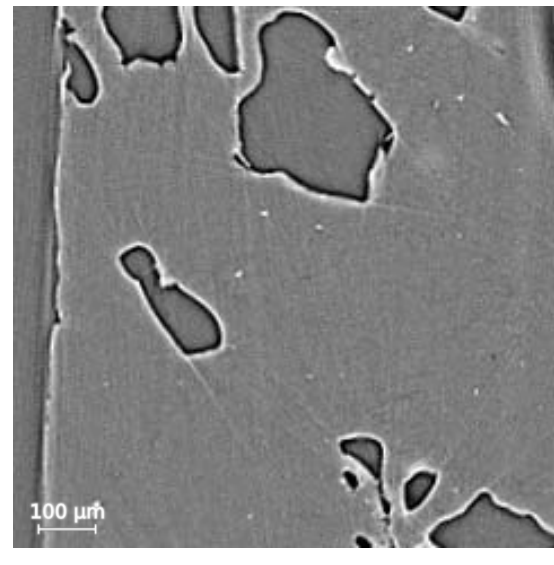

(a) in air

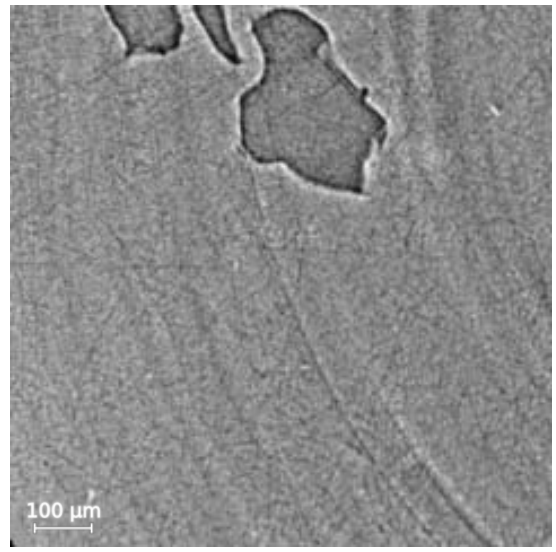

(b) under 104 bars

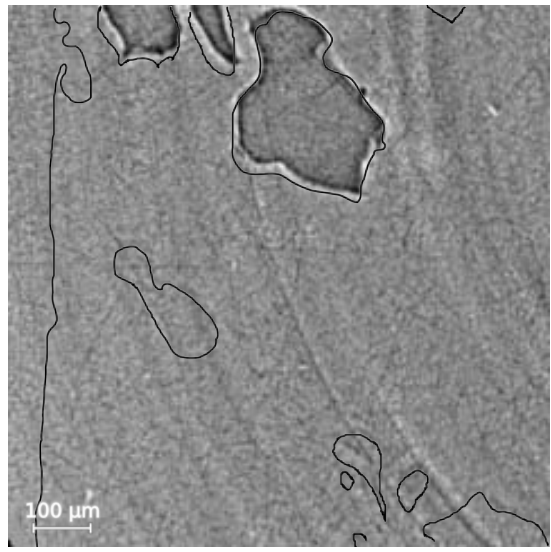

(c) under 104 bars with mask

Figure 5: filling in of porosities in the MPP foam.

To show where the first disappearing pores are located a Z-projection of the thresholded stacks was calculated. Figure $6 \mathrm{a}$ shows the initial state and $6 \mathrm{~b}$ the 104 bars state. In these two figures, the lighter regions represent the presence of porosities. It is clear from these two pictures that the missing porosities at 104 bars are located close to the edges of the sample. To highlight this further, figure $6 \mathrm{c}$ shows the difference between figure $6 \mathrm{a}$ and figure $6 \mathrm{~b}$. In this latter image, the lighter pores are those having disappeared between the two images. Again these are clearly observed to be located at the periphery of the sample. We believe that this happens because the applied pressure progressively breaks the connecting walls between cavities touching the surface and more interior ones.

In the case of the PP foam, an other mechanism is observed: the size of the cavities decreases. One illustration of this is shown for one pore in table 4 . The volume of the selected pore decreases with pressure. It loses $8.65 \%$ of its initial volume from 0 to 104 bars of pressure. This was suspected from the qualitative observation in figure 5 and is supported by the measurement here.

\section{Quantitative analysis}

\subsection{Image processing procedure}

Three phases coexist in a syntactic foam: gas, glass and polymer. As the scan resolution was $3.5 \mu \mathrm{m}$ and the thickness of the glass was around $1.5 \mu \mathrm{m}$, we consider only two phases: gas and the rest. In the case of foamed PP, there are obviously only two phases. The contrast between the ethanol and the polymer is almost nonexistent. Because of this problem, we decided to create a binary mask from the first state. This mask allow us to find the outline for the edges of the sample in the other states. But in the case of the GSPU 


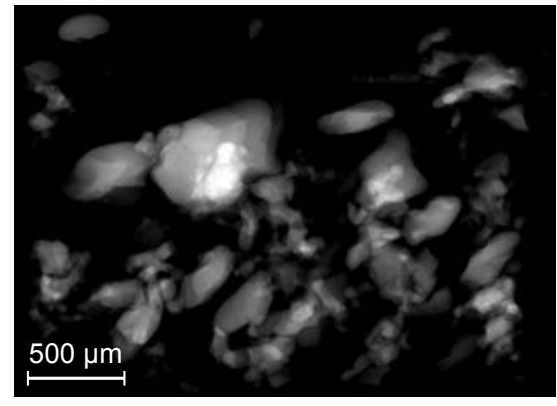

(a) initial state

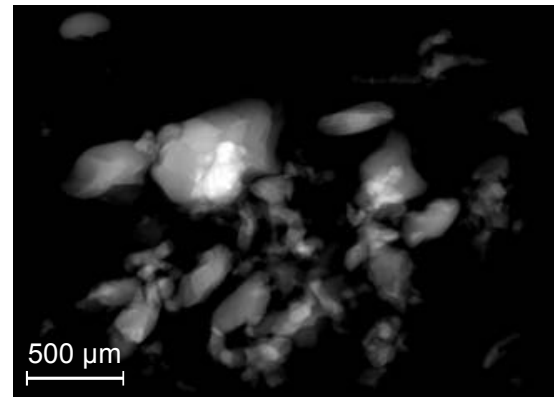

(b) after 104 bars

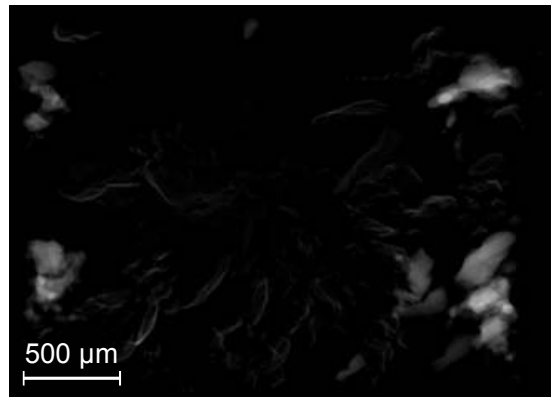

(c) difference of the two states

Figure 6: X-ray transmission seen from the top of the sample constructed with a Z-projection of the thresholded blocs.

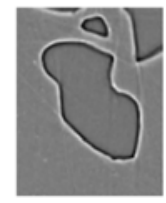

\begin{tabular}{lrr}
\hline Step & Volume of the porosity & Porosity reduction \\
\hline In air & $102.55 \times 10^{5} \mu \mathrm{m}^{3}$ & $0.00 \%$ \\
In ethanol & $102.28 \times 10^{5} \mu \mathrm{m}^{3}$ & $0.27 \%$ \\
Under 55 bars & $98.02 \times 10^{5} \mu \mathrm{m}^{3}$ & $4.42 \%$ \\
Under 104 bars & $93.68 \times 10^{5} \mu \mathrm{m}^{3}$ & $8.65 \%$ \\
\hline
\end{tabular}

Table 4: loss of volume for a pore of the MPP foam.

matrix as shown in figure 4, the sample volume increases, so we decided to do a 3D-directional dilatation of the mask to coincide with the dilation observed in figure 4 . The position of the sample after putting it into the chamber is difficult to adjust, so this is achieved by rotating numerically (using ImageJ [20]) and after reconstruction the scanned block to coincide with the position of the sample in the initial state. Finally the block was thresholded to obtain the gas phase.

\subsection{Evolution of the porositiy}

Figure 7 shows the percentage of evolution in the volume of porosity $V(p)$ divided by its initial volume $V_{0}$ (before dipping the sample in ethanol) for each material when the pressure $p$ is increased. The triangle represents the initial state $(100 \%)$.

Dipping the sample into ethanol leads to a decrease in porosity for the three samples. This is mainly due to the filling of porosities connected to the surface of the sample. The decrease in the GSPP foam amounts to $\sim 2 \%$, while for the GSPU it is about $10 \%$. The high ethanol uptake of the PU matrix may help the ethanol to fill in the broken microsphere, on the contrary to the situation observed in the case of a poor ethanol uptake of the PP matrix. Regarding the foamed PP, $18 \%$ of the porosity disappears when it is immersed in ethanol. This is probably the result of micro-cracks connecting the surface and the porosity close to the surface. The MPP loses $27 \%$ of its porosity when the pressure is increased to 133 bars. This is much more that what has been measured in table 4 on the compression of one observed single pore. Again we believe that this is due to the filling rather than volume compression, because small bridges between cavities are progressively broken due to the applied pressure and leads to a propagation of the liquid inside the porosity from the cavities at the surface towards inner cavities.

\subsection{Profiles of gas fraction in slices}

Three orthogonal slices (perpendicular to the three principal directions which are the rotation axis $z$, the beam direction $x$ at $0^{\circ}$ and the beam direction $y$ at $90^{\circ}$ ) can be used to highlight the porosity distribution in 


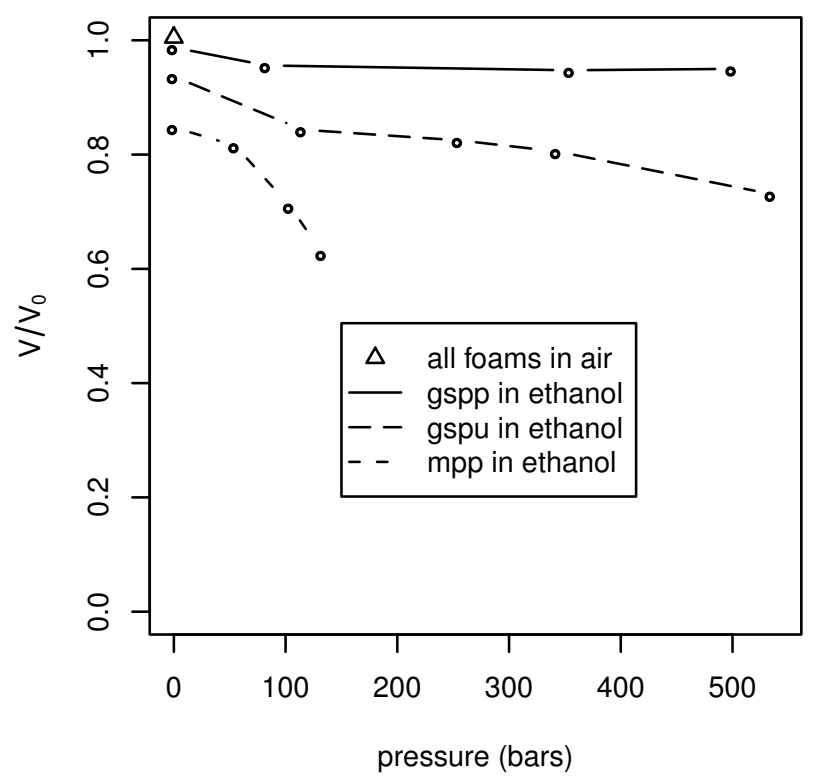

Figure 7: $V / V_{0}$ is the evolution of the porosity in three foams during loading where $V_{0}$ represent the initial volume of porositiy in the air and $V$ the volume of porositiy in ethanol and under pressure.

the sample as a function of the applied pressure. The fraction of porosity can be measured in each of these slices and then plotted as a function of the distance between the slice and the edge of the sample. This allows us to plot the porosity profiles shown in the next sections. For the sake of clarity, only the profiles in slices perpendicular to the $x$ direction are shown in this paper but it has been verified that the other types of profiles (perpendicular to $y$ or $z$ ) convey the same sort of information.

The next figures (8,9 and 10) show the profiles of gas fraction in slices in the $x$ directions in the foam. The profiles shown are the initial state, the ethanol immersed state (black line), the ethanol immersed and some of the pressure states.

\subsubsection{Foam GSPP}

The quasi non-evolution of the GSPP is shown again in these graphs. This supports figure 7 where it was shown that the average amount of porosity changes only slightly when the pressure increases. The graphs show however that the loss of porosity is located at the edges of the sample.

\subsubsection{Foam GSPU}

In the initial state (solid line style), the edges of the sample are located at $x=0.2$ and $x=2.35$. But in the next step, these edges moved to be $x=0$ and $x=2.5$. This displacement is the displacement of the microspheres due to the swelling of the matrix. Between the states in air and in ethanol, the decrease of the gaz fraction is more important at $x=0$ than at $x=2.5$. As explained in paragraph 4.1 and shown in figure $4 \mathrm{a}$, the edge at $x=2.5$ was initially in contact with the mould and so has less void in contact with the surface. Away from the surfaces the decrease of gas fraction is quite isotropic.

\subsubsection{Foam $M P P$}

Gas fraction profiles are more difficult to comment in this case because of the size of the porosities compared to the size of the sample. No change in the amount of porosity is observed when the sample is dipped into ethanol. It can be seen that at this scale, spatial distribution of the porosity is poor compared 


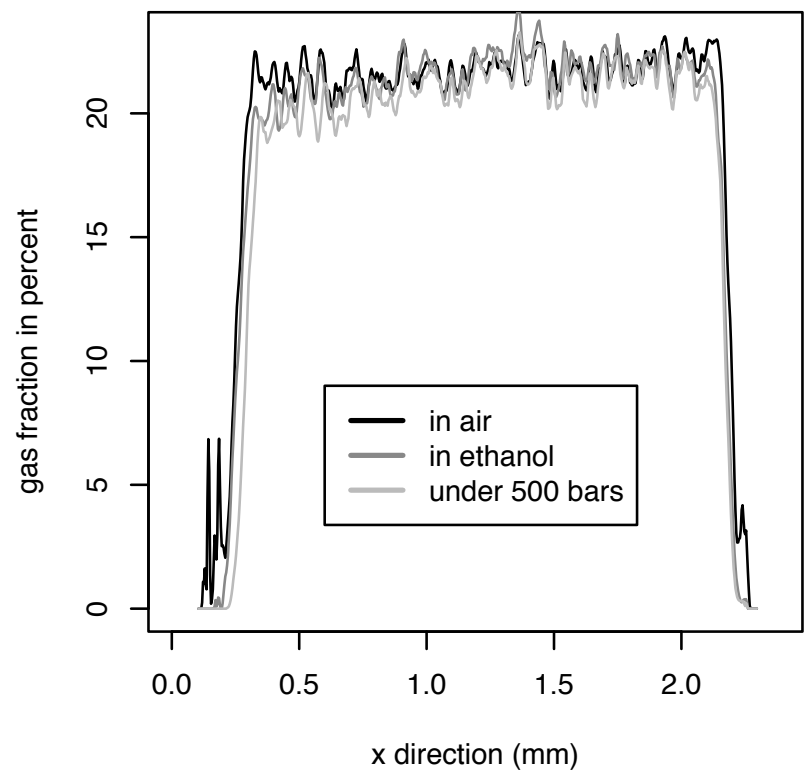

Figure 8: comparison of gas fraction profile along the $x$ direction at different steps in GSPP.

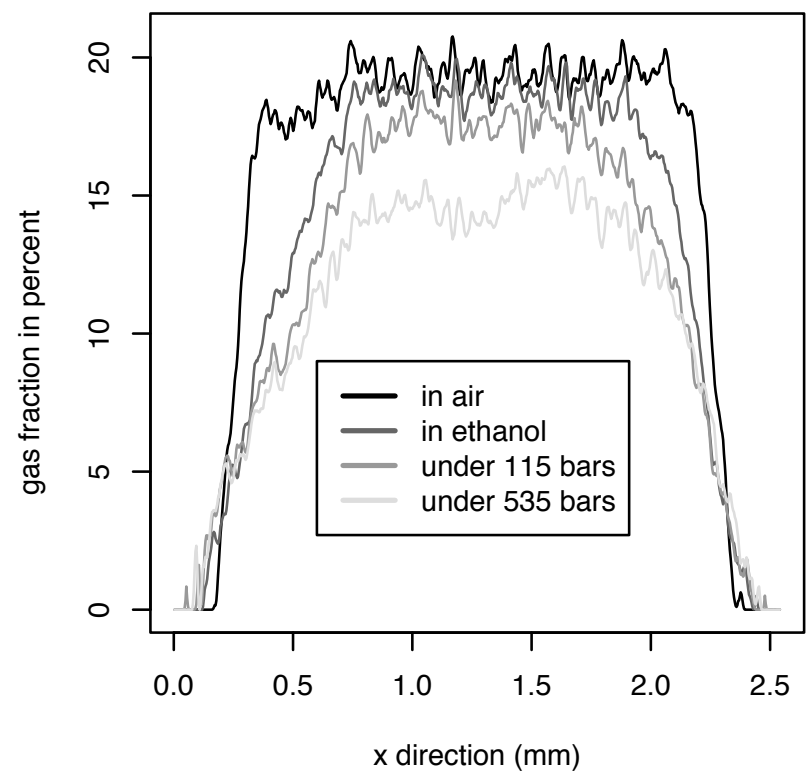

Figure 9: comparison of gas fraction profile along the $x$ direction at different steps in GSPU. 


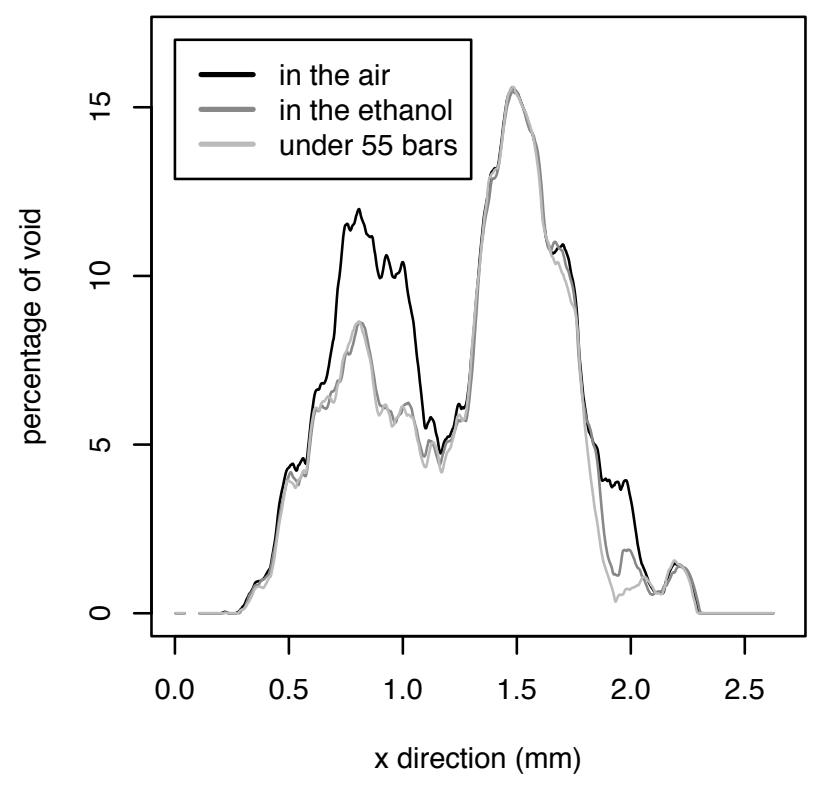

Figure 10: comparison of gas fraction profile along the $x$ direction at different steps in MPP.

to the small fluctuation observed in the two preceding graphs. When the pressure is increased, and when a porosity fills in, it leads to a great variation in the profile.

\section{Discussion}

Two main mechanisms are observed in the present study. The first is the swelling of the polymeric matrix due to ethanol absorption. This is very spectacular in the polyurethane matrix but doesn't seem to occur in the polypropylene matrix. The polymer fluid absorption is known [13, 15, 21]. It is also shown in [21], that swelling can be observed with water rather than ethanol. In his article on hydrostatic compression of PP foams [15] Philippe Viot solved the water absorption problem by coating the samples with a silicone gel which protects from water penetration.

The second striking mechanism observed in this study is the filling of the porosities by a fluid. Our observation technique is not capable of distinguishing whether this fluid is the polymeric matrix itself or ethanol as both fluids absorb the X-rays in the same manner. In the case of the syntactic foams GSPP and GSPU, the fluid penetrates the matrix little by little and fills in the microspheres. We observed however that in the case of the PP matrix, the filling process can be rather slow (filling of a same sphere observed over several scans). This might indicate that the fluid is more viscous in this case. This probably concerns those microspheres that are damaged either before or due to the application of hydrostatic pressure. Note that in both cases, this would lead to the measurement of an apparent volume reduction of the sample as it is observed at a macroscopic level when these samples are tested under isostatic pressure. It should also be noted that this observation is very different than the internal fragmentation of microspheres observed on similar samples during dry compression constrained in a cylinder [16].

If the observed filling fluid is ethanol, it can come either from the matrix or by diffusion at the matrix/spheres interfaces. We have shown that the Polyurethane matrix probably contains a lot of ethanol as illustrated by the swelling of the sample. Under pressure, this ethanol can then possibly fill in the broken spheres. Polypropylene, on the contrary, is very stable and compatible with ethanol and we confirmed that 
no swelling is observed in this case. So it seems rather difficult to imagine that ethanol could be absorbed in the matrix but we can not reject its diffusion along the matrix/spheres interfaces. In the material studied, the spheres are percolating so this network of interfaces can form a path leading to the middle of the sample. This infiltration mechanism might then be an artefact of the small size of the samples used in this study. The sample being small, each sphere is always rather close to the pressurized liquid so it might be possible for this liquid to flow. For both materials, it is also possible that what we see is not infiltration of the spheres but polymeric matrix. Confirming this would require further experimentation.

It could be interesting to conduct the same sort of studies with a silicone gel coating around the sample as in [15] but this is beyond the scope of the present paper. As for the MPP material, we observed that the pores close to the surface fill up with ethanol when dipped in the fluid. Subsequently when the pressure is increased, more pores also fill with ethanol, possibly because the pressure breaks the separation between the already infiltrated pores and their neighbours towards the centre of the sample.

\section{Conclusions}

The compression behaviour of syntactic polymer foams reinforced or not with hollow glass spheres has been studied during hydrostatic compression experiments coupled with high-resolution X-ray tomography observation using synchrotron radiation. Three different materials have been selected for the study, two of them having approximately the same kind of microspheres but differing in terms of matrix material, and the last being only a foamed polymer with no spheres.

In foam GSPP and MPP the matrix was an elasto-plastic PP and in foam GSPU an elastomeric PU. The 3D images were processed and the morphology of microspheres in the initial stage, immersed in ethanol and compressed states was analysed for the three materials. The samples were not in an impermeable enclosure. The principal mechanism shown during the different steps is the progressive migration of a fluid into the matrix accelerated by the compression. The PP matrix seems to be more resistant to ethanol uptake than the PU matrix. In the GSPU foam, the migration of the fluid has for direct consequence, the swelling up of the matrix. Once the fluid has penetrated into the matrix, it fills in the porosities which are the closest to the edges when there is no enclosure around the gas (like in MPP) and where the microspheres are broken. The broken fragments of the spheres remained attached to the matrix, what we observed being just a replacement of the gas by a fluid. We can not conclude if this fluid is ethanol or the matrix itself. We suspect that in the case of the GSPU matrix, swollen with ethanol, and in the case of the MPP material, what we observe is the ethanol filling of the cavities. In the case of the GSPP material, matrix intrusion inside the hollow spheres is also a possibility.

\section{References}

[1] Franklin, J.F., Wright, A.. In: BHR limited. Edinburgh, UK; 1999,.

[2] Watkins, L., Hershey, E.. In: World Pipelines; vol. 4. 2004, p. 49.

[3] Van Belle, B.. In: Offshore technology conference. 14120; Houston, TX, USA; 2002,.

[4] Song, B., Chen, W., Yanagita, T., Frew, D.J.. Composite Structures 2005;67:279-287.

[5] Wouterson, E.M., Boey, F.Y., Hu, X., Wong, S.C.. Composites Science and Technology 2005;65:1840-1850.

[6] Kim, H.S., Plubrai, P.. Composites Part A: Applied Science and Manufacturing 2004;35:1009-1015.

[7] Rizzi, E., Papa, E., Corigliano, A.. International Journal of Solids and Structures 2000;37:5773-5794.

[8] Gupta, N., Woldesenbet, E.. Composite Structures 2003;61:311-320.

[9] Bardella, L., Genna, F.. International Journal of Solids and Structures 2001;38:7235-7260.

[10] Grosjean, F., Bouchonneau, N., Choqueuse, D., Sauvant-Moynot, V.. Journal of Materials Science 2009;44:1462-1468.

[11] Choqueuse, D., Davies, P., Perreux, D., Sohier, L., Cognard, J.Y.. Applied Mechanics and Materials 2010;24-25:97-102.

[12] Bouchonneau, N., Sauvant-Moynot, V., Choqueuse, D., Grosjean, F., Poncet, E., Perreux, D.. Journal of Petroleum Science and Engineering 2010;73:1-12.

[13] Gimenez-Fouque, N.. Vieillissement hydrolique de mousses syntactiques époxyde/amine/verre pour l'isolation thermique sous hautes pressions : Mécanismes de dégradation et simulation de la prise en eau. Ph.D. thesis; Institut National de Sciences Appliquées de Lyon; 2005.

[14] Maire, E., Gimenez, N., Sauvant-Moynot, V., Sautereau, H.. Philos Transact A Math Phys Eng Sci 2006;364:69-88.

[15] Viot, P.. International Journal of Impact Engineering 2009;36:975-989.

[16] Adrien, J., Maire, E., Gimenez, N., Sauvant-Moynot, V.. Acta Materialia 2007;55:1667-1679. 
[17] Buffiere, J.Y., Maire, E., Adrien, J., Masse, J.P., Boller, E.. Experimental Mechanics 2010;50:289-305.

[18] Maire, E., Buffière, J.Y., Salvo, L., Blandin, J.J., Ludwig, W., Létang, J.M.. Advanced Engineering Materials 2001;3:539-546.

19] Takiguchi, Y., Uematsu, M.. The Journal of Chemical Thermodynamics 1996;28:7-16.

[20] Schneider, C.A., Rasband, W.S., Eliceiri, K.W.. Nat Methods 2012;9:671-675.

[21] Braun, J., Klein, M.O., Bernarding, J., Leitner, M.B., Mika, H.D.. Polymer Testing 2003;22:761-767. 